\section{Meteoritics as petrography}

\author{
John A. Wood
}

Meteorites: A Petrologic-Chemical Synthesis. By Robert T. Dodd. Pp.432. ISBN 0-521-22570-1. (Cambridge University Press: 1982.) £35, \$69.50.

METEORITES are complex, ancient, enigmatic rock fragments from somewhere beyond Mars. They were formed at the same time as the Solar System, and they retain a cryptic record of the events of that time. In the 30 years since Harold Urey and Harrison Brown unleashed the techniques and concepts of post-War geochemistry on these objects, meteoritics has burgeoned into an important scientific subdiscipline. Many studies have been carried out and as many papers published; a number of important insights have been gained; and several riddles are still with us, more prickly and intractable than ever.

The field has always needed a good reference book, the more so as its literature has expanded to a scale greater than any one person can assimilate. Several books about meteorites have been written at a professional level in the past decade, but none can be said to fill the need. Robert Dodd's Meteorites: A PetrologicChemical Synthesis is the latest candidate. It is important to include the subtitle, because the emphasis of the book is very strongly on the petrology (or, to be more precise, the petrography) and chemistry of meteorites, Dodd's own speciality. (Petrography is the descriptive study of the microstructures and component mineralogies of rocks.)

Many good things can be said about the book. Dodd writes in a readable, straightforward style. His coverage of petrography and chemistry is comprehensive, scholarly and exhaustively referenced. Material is rationally organized, most of the chapters dealing with discrete subclasses of meteorites. (Two exceptions are a chapter on radiometric studies of meteorites, and one on the asteroid-sized parent bodies that they once resided in.) He strikes an appropriate balance between descriptions and interpretive discussions. In the latter, he is as objective as can reasonably be expected of a mortal in this contentious field. Each chapter is preceded by a précis that maps out for the reader the ground to be covered. There is an excellent index, and the illustrations are more than adequate in number and quality.

However, I fault the book for presenting a narrow view of meteoritics, that is, one largely limited to petrographic and geochemical studies. Perhaps it is unreasonable to expect Dodd, or anyone, to cover the whole marvellously diverse field of meteoritics as comprehensively as the author has treated meteoritic petrography and chemistry. The book would be twice as long and would cost Dodd far more than twice the labour, since he would have to venture beyond his field of experience.

But the book as it stands unfortunately presents the study of meteorites as an end in itself rather than as a means to a greater end: an understanding of the origin of the Solar System. The stage on which this drama occurred was the solar nebula, a giant accretion disk of gas and dust where the properties of the meteorites and, ultimately, the planets, were established. Dodd cannot avoid making repeated references to the nebula, the context in which everything happened; but he eschews any systematic discussion of astrophysical models of the nebula because they are "varied and changeable". Lacking such a framework, he cannot put the properties of meteorites to work in clarifying our view of the nebula.

"Nebula" does not appear in the index of the book; nor does "Solar System, origin"; nor does "FUN inclusions". The latter, the subject of intensive isotopic research which gives us a fascinating view of pre-Solar System events, are touched on only momentarily, and then not by the name "FUN". It is regrettable that the non-petrographic aspects of meteorites are given such short shrift: the most inspiring aspects of meteoritics are the grand vista it examines, and the close collaboration of researchers from many diverse disciplines in attacking the problem. Meteorites: $A$ Petrologic-Chemical Synthesis exposes only a near-sighted and parochial view of the goals of this field. It is, however, a useful and excellent reference work within the unfortunately restrictive boundaries it has erected.

John A. Wood is an Associate Director of the Harvard-Smithsonian Center for Astrophysics, Cambridge, Massachusetts.

\title{
Welcome to theoretical particle physics
}

\section{P.V. Landshoff}

Gauge Theories in Particle Physics: $A$

Practical Introduction. By Ian J.R. Aitchison and Anthony J.G. Hey. Pp.341. Pbk ISBN 0-85274-534-6. (Adam Hilger/ Heyden: 1982.) £14.50, \$36.

THERE are still very few books on the topics that now excite the interest of particle theorists - the electroweak theory that brings together electromagnetism and the weak nuclear interaction, and quantum chromodynamics, the candidate theory of the strong nuclear interaction. So this book, which sets out to be a compact introduction to these subjects, will be warmly welcomed, especially for the attention it gives to the distinctive properties of the gauge symmetry underlying the new theories.

Quantum electrodynamics is the prototype gauge theory and is described by Aitchison and Hey as "the best theory we have"'. Its best test is the magnetic moment of the electron, whose calculated and measured values agree to within one part in $10^{10}$. Electrodynamics is a gauge theory because its physical predictions are invariant under certain transformations of the electromagnetic potentials. Only this gauge invariance makes it possible to remove unphysical infinities from the quantized version of the theory (renormalization).

Quantum electrodynamics is based on Maxwell's equations, the first description of the electric and the magnetic force in the same set of equations. A century later, Glashow, Salam and Weinberg enlarged the theory so as to incorporate the weak interaction (responsible, for example, for neutron $\beta$-decay). Their unified electro- weak gauge theory is steadily accumulating an impressive list of successes.

Another gauge theory, chromodynamics, seems to give a good description of the nuclear force. Attempts to unify this with the electroweak theory have led to the prediction that protons are unstable, with a lifetime of about $10^{31}$ years. Four candidate proton-decay events have recently been observed, but the data are not yet a sufficient test.

Quantum electrodynamics itself is far from easy, but the electroweak theory and chromodynamics are less accessible to most physicists because the groups of gauge transformations are not Abelian (which implies that the underlying gauge transformations do not necessarily commute with each other). This book sets out to make the theories as simple as possible. Everything is here, from the elements of relativistic quantum mechanics to a mention of proton decay in grand unified models, with renormalization and even a brief discussion of the quark-parton model on the way.

Inevitably in a short book of wide scope, nothing is treated very fully. In places, plausibility arguments replace proper derivations. But experimentalists especially will find it helpful in providing an understanding of the essential ideas behind what the theorists are talking about, while neophyte theorists will find it a valuable introduction to the more advanced texts. The second half of the book will be the most useful, for the painless insight that it gives into non-Abelian physics.

Peter Landshoff is Reader in Mathematical Physics at the University of Cambridge. 